\title{
Effects of respiratory mechanics on the capnogram phases: importance of dynamic compliance of the respiratory system
}

\author{
Barna Babik ${ }^{1}$, Zsófia Csorba', Dorottya Czövek², Patrick N Mayr ${ }^{3}$, Gábor Bogáts $^{4}$ and Ferenc Peták²*
}

\begin{abstract}
Introduction: The slope of phase III of the capnogram (S $S_{\text {III }}$ relates to progressive emptying of the alveoli, a ventilation/perfusion mismatch, and ventilation inhomogeneity. $S_{\text {III }}$ depends not only on the airway geometry, but also on the dynamic respiratory compliance (Crs); this latter effect has not been evaluated. Accordingly, we established the value of $S_{\| I}$ for monitoring airway resistance during mechanical ventilation.

Methods: Sidestream capnography was performed during mechanical ventilation in patients undergoing elective cardiac surgery $(n=144)$. The airway resistance (Raw), total respiratory resistance and Crs displayed by the ventilator, the partial pressure of arterial oxygen $\left(\mathrm{PaO}_{2}\right)$ and $\mathrm{S}_{\text {III }}$ were measured in time domain $\left(\mathrm{S}_{\mathrm{T}-\mathrm{III}}\right)$ and in a smaller cohort $(n=68)$ by volumetry $\left(\mathrm{S}_{\mathrm{V}-\mathrm{II}}\right)$ with and without normalization to the average $\mathrm{CO}_{2}$ phase III concentration. Measurements were performed at positive end-expiratory pressure (PEEP) levels of 3,6 and 9 $\mathrm{CmH}_{2} \mathrm{O}$ in patients with healthy lungs (Group HL), and in patients with respiratory symptoms involving low (Group LC), medium (Group MC) or high Crs (Group HC).

Results: $\mathrm{S}_{\mathrm{T}-\mathrm{III}}$ and $\mathrm{S}_{\mathrm{V}-\mathrm{II}}$ exhibited similar PEEP dependencies and distribution between the protocol groups formed on the basis of Crs. A wide interindividual scatter was observed in the overall Raw-S $\mathrm{S}_{\text {T-III }}$ relationship, which was primarily affected by Crs. Decreases in Raw with increasing PEEP were reflected in sharp falls in $\mathrm{S}_{\text {III }}$ in Group HC, and in moderate decreases in $\mathrm{S}_{\| I}$ in Group MC, whereas $\mathrm{S}_{T-\text { III }}$ was insensitive to changes in airway caliber in Groups $\mathrm{LC}$ and $\mathrm{HL}$.

Conclusions: $S_{|| \mid}$assessed in the time domain and by volumetry provide meaningful information about alterations in airway caliber, but only within an individual patient. Although $\mathrm{S}_{\mathrm{T}-\text { III }}$ may be of value for bedside monitoring of the airway properties, its sensitivity depends on Crs. Thus, assessment of the capnogram shape should always be coupled with Crs when the airway resistance or oxygenation are evaluated.
\end{abstract}

\section{Introduction}

Capnography is a noninvasive, continuous, online, dynamic, effort- and cooperation-independent method for bedside monitoring of the exhaled carbon dioxide $\left(\mathrm{CO}_{2}\right)$ concentration. The slope of the third phase of the capnogram $\left(\mathrm{S}_{\mathrm{III}}\right)$ is determined physiologically by the continuous $\mathrm{CO}_{2}$ excretion from the pulmonary vasculature and the periodic lung ventilation $[1,2]$, and the interactions between the diffusive and convective gas mixing [3-5]. The development of pathophysiological

\footnotetext{
* Correspondence: petak.ferenc@med.u-szeged.hu

${ }^{2}$ Department of Medical Physics and Informatics, University of Szeged, 9

Korányi fasor, H-6720 Szeged, Hungary

Full list of author information is available at the end of the article
}

ventilatory and/or perfusion inhomogeneities in time and space leads to lung compartments containing various $\mathrm{CO}_{2}$ concentrations, and this may further elevate $\mathrm{S}_{\text {III }}[2,6-13]$.

The sensitivity of $\mathrm{S}_{\text {III }}$ to ventilation/perfusion abnormalities suggested its clinical usefulness for the detection of respiratory abnormalities or the following of ventilatory and/or pharmacological interventions. Numerous studies have demonstrated that the magnitude of $\mathrm{S}_{\mathrm{III}}$ reflects the severity of emphysema or asthma [4,11,13-17], cystic fibrosis and bronchiectasis [8], chronic obstructive pulmonary disease (COPD) [7], chronic bronchitis [9] and acute lung injury $[6,10]$. Inconsistent associations have been reported in previous attempts to clarify the quantitative relationships 
between capnographic and lung function indices. Earlier studies reported a strong correlation between the forced expiratory volume in one second $\left(\mathrm{FEV}_{1}\right)$ and $\mathrm{S}_{\mathrm{III}}[15]$, merely a modest association $[11,16]$, or even a lack of correspondence [13]. Furthermore, significant correlations were observed between the total respiratory resistance (Rrs) and $\mathrm{S}_{\mathrm{III}}$ in mechanically ventilated patients, however $\mathrm{S}_{\text {III }}$ had limited clinical applicability to predict Rrs [18]. Thus, in consequence of the complex mechanisms affecting $\mathrm{S}_{\mathrm{III}}$, its diagnostic and/or monitoring value is far from being clear. The diverse emptying of different lung compartments with various $\mathrm{CO}_{2}$ levels is determined not only by the airway geometry, but also by the driving pressure governed by the dynamic respiratory compliance (Crs), including the chest wall and the lung. Despite the obvious importance of respiratory tissue elastance in determining the expiratory flow and the rate of $\mathrm{CO}_{2}$ clearance, the role of the respiratory elastic recoil on the capnogram shape has not been examined to date.

The aim of the present prospective consecutive clinical study was to investigate systematically whether the capnogram shape is affected by changes in both airway caliber and the Crs in mechanically ventilated patients. We also set out to clarify the contribution of the altered airway properties and tissue mechanics with increasing positive end-expiratory pressure (PEEP) to the changes in $\mathrm{S}_{\text {III }}$. To test the hypothesis that both the airway geometry and the Crs reflecting the elastic recoil of the respiratory system affect $\mathrm{S}_{\mathrm{III}}$, a large cohort of mechanically ventilated patients with normal and diseased lungs was studied.

\section{Materials and methods Patients}

One hundred and forty-four patients (93 males, 51 female, $62 \pm 9($ mean \pm SD) years of age (range 39 to 84 years) undergoing elective coronary bypass surgery were examined in the supine position before the surgical procedure. The protocol was approved by the Human Research Ethics Committee of Szeged University, Hungary (no. WHO 2788), and the patients gave their informed consent to the study. The patients were premedicated with intramuscular morphine $(0.07 \mathrm{mg} / \mathrm{kg})$ and midazolam $(0.07 \mathrm{mg} / \mathrm{kg}) 1 \mathrm{~h}$ before the operation. Anesthesia was induced with i.v. midazolam $(30 \mu \mathrm{g} / \mathrm{kg})$, sufentanil $(0.4$ to $0.5 \mu \mathrm{g} / \mathrm{kg})$ and propofol ( 0.3 to $0.5 \mathrm{mg} / \mathrm{kg})$. Muscle paralysis was achieved with an i.v. bolus of rocuronium $(0.6 \mathrm{mg} / \mathrm{kg})$. The anesthesia and muscle relaxation were maintained with i.v. infusions of propofol $(50 \mu \mathrm{g} / \mathrm{kg} / \mathrm{min})$ and i.v. boluses of rocuronium $(0.2 \mathrm{mg} / \mathrm{kg}$ every $30 \mathrm{~min})$.

The trachea was intubated with a cuffed endotracheal tube with an internal diameter of 7,8 or $9 \mathrm{~mm}$, and the patients were ventilated with a Dräger Zeus anesthesia machine (Lübeck, Germany) in volume-controlled mode with descending flow. The ventilator frequency was set to 12 to 14 breaths $/ \mathrm{min}$, a tidal volume of $7 \mathrm{ml} / \mathrm{kg}$ and a PEEP of $3 \mathrm{cmH}_{2} \mathrm{O}$ were applied. The fraction of inspired oxygen $\left(\mathrm{FiO}_{2}\right)$ was maintained at 0.5 throughout the entire study period. Arterial blood gas samples were analyzed at least hourly (Radiometer ABLTM 505, Copenhagen, Denmark). The ejection fraction (EF) data were collected from preoperative echocardiography. The body mass index (BMI) of each patient was calculated.

The patients had various cardiac diseases including ischemic heart disease $(n=108)$, a mitral insufficiency $(n=21)$, aortic stenosis $(n=38)$, and other types of cardiac malformation $(n=5)$, such as myxoma, congenital heart disease or aortic aneurysm. The patients exhibited wide variations in their pulmonary status, with some of them having no pulmonary symptoms (that is, no history of lung disease, normal BMI, no pleural effusion, no pulmonary congestion, no smoking history, lack of wheezing periods within the past six months, lack of use of bronchodilator drugs; $n=29$ ). Others had lung abnormalities causing restrictive (pulmonary congestion $(n=45)$ or obesity (BMI $\geq 31 ; n=48)$ ) and/or obstructive changes (emphysema $(n=55)$, asthma $(n=14)$, chronic bronchitis $(n=25)$ or sarcoidosis $(n=1))$.

To establish whether the elastic properties of the respiratory system affected the capnogram shape, the patients with respiratory symptoms were allocated into three groups, on the basis of their Crs. This Crs was determined $10 \mathrm{~min}$ after anesthesia induction and a lung volume homogenization maneuver (that is, lung inflation and maintenance at a transrespiratory pressure of 30 $\mathrm{cmH}_{2} \mathrm{O}$ for $5 \mathrm{~s}$ ) when stable hemodynamic and ventilatory conditions at PEEP $3 \mathrm{cmH}_{2} \mathrm{O}$ have been reached (that is, prior to the first capnogram recording). Group LC comprised patients with $\mathrm{Crs}$ in the lowest tenth percentile $\left(\mathrm{Crs}<34.5 \mathrm{ml} / \mathrm{cmH}_{2} \mathrm{O} ; n=15\right)$, and Group $\mathrm{MC}$ patients with $\mathrm{Crs}$ between the tenth and the ninetieth percentile $\left(34.5<\mathrm{Crs}<69 \mathrm{ml} / \mathrm{cmH}_{2} \mathrm{O} ; n=85\right)$, and Group $\mathrm{HC}$ patients with $\mathrm{Crs}$ above the ninetieth percentile $\left(\mathrm{Crs}>69 \mathrm{ml} / \mathrm{cmH}_{2} \mathrm{O} ; n=15\right)$. Patients with healthy lungs were regarded as an independent group (Group HL; $n=29$ ). The patients were classified based on the Crs measured after a lung volume recruitment maneuver. The characteristics of the patients in each protocol group are summarized in Table 1 . The age of the patients did not differ significantly in the different groups $(P=0.16)$.

\section{Measurement of airway and respiratory tissue mechanics}

Details of the measurement of the input impedance of the respiratory system ( $\mathrm{Zrs}$ ) were reported previously [19]. Briefly, a T-piece with two collapsible segments was attached to the distal endotracheal tube, with one end connected to the respirator and the other end to a loudspeaker-in-box system. This apparatus allowed switching 
Table 1 Number of patients with different conditions/diagnoses in each protocol group.

\begin{tabular}{lcccc}
\hline & Gender* $\left.^{*} \mathbf{m} / \mathbf{f}\right)$ & Obesity* $^{*}(\mathbf{l o w} / \mathrm{ob})$ & Pulmonary status E*/A*/CB*/OLD & Cardiac disease CAD/AS/MI/LEF*/OCD \\
\hline Group HL $(\boldsymbol{n}=\mathbf{2 9})$ & $20 / 9$ & $10 / 19 /-$ & $-/-/-/-$ & $26 / 3 / 3 /-/ 1$ \\
\hline Group HC $(\boldsymbol{n}=\mathbf{1 5})$ & $15 /-$ & $8 / 7 /-$ & $14 /-/ 6 /-$ & $11 / 4 /-/-/-$ \\
\hline Group MC $(\boldsymbol{n}=\mathbf{8 4})$ & $56 / 28$ & $12 / 28 / 44$ & $37 / 10 / 15 / 2$ & $61 / 28 / 8 / 10 / 3$ \\
\hline Group LC $(\boldsymbol{n}=\mathbf{1 5})$ & $3 / 12$ & $-/ 5 / 10$ & $4 / 4 / 4 /-$ & $10 / 3 / 1 / 8 / 1$ \\
\hline
\end{tabular}

Obesity categories: $\mathrm{n}$, normal $(20 \leq \mathrm{BMI}<25)$; ow, overweight $(25 \leq \mathrm{BMI}<30)$; ob, obese $(30 \leq \mathrm{BMI})$. Pulmonary status: $\mathrm{E}$, emphysema; $\mathrm{A}$, asthma; CB, chronic bronchitis; OLD, other lung disease. Cardiac disease: CAD, coronary artery disease; AS, aortic stenosis; MI, mitral insufficiency; LEF, low ejection fraction (EF < $50 \%) ; O C D$, other cardiac disease. Pulmonary and cardiac conditions are based on previous clinical diagnoses. ${ }^{*}, P<0.05$ between the expected and the observed frequencies in the protocol groups for each variable. Group HC, group of patients with high dynamic respiratory compliance; Group $\mathrm{HL}$, group of patients with healthy lungs; Group LC, group of patients with low dynamic respiratory compliance; Group MC, group of patients with medium dynamic respiratory compliance.

of the patient from the respirator to the forced oscillatory setup during the recordings. These were performed by generating pseudorandom pressure excitations into the trachea during short (15 s) apneic pauses superimposed into the mechanical ventilation. The forcing signal contained 30 integer-multiple components of the $0.2 \mathrm{~Hz}$ fundamental frequency, in the frequency range 0.2 to $6 \mathrm{~Hz}$. Tracheal airflow ( $\left.\mathrm{V}^{\prime}\right)$ was measured with a $28 \mathrm{~mm}$ ID screen pneumotachograph connected to a differential pressure transducer (ICS model 33NA002D; ICSensors, Milpitas, CA, USA). The airway opening pressure (Pao) was detected with an identical pressure transducer. Zrs was computed from the power spectra of Pao and V', and then ensemble-averaged under each condition. The mean Zrs data were fitted by a well-validated four-parameter model [20] containing a frequency-independent airway resistance (Raw) and inertance (Iaw) and a constantphase tissue compartment characterized by the coefficients of damping $(\mathrm{G})$ and elastance $(\mathrm{H})$.

\section{Recording and analyses of the capnogram}

Changes in partial $\mathrm{CO}_{2}$ pressure in the exhaled gas during mechanical ventilation were measured with a calibrated sidestream capnometer (Ultima ${ }^{\mathrm{TM}}$, Datex/Instrumentarium, Helsinki, Finland). Since capnograms are displayed in clinical routine in the time domain, time capnography was applied in each patient to record $\mathrm{CO}_{2}$ changes. To minimize the possible drawback of this time domain analyses, we paid attention to involve only the linear part of the $\mathrm{CO}_{2}$ trace in the readings of $\mathrm{S}_{\mathrm{III}}$. Nevertheless, volumetric capnography may allow a better distinction between the phases $[2,4,6,7,10,12,14,21]$ and thus, in a subgroup including the last 68 patients, the flow during mechanical ventilation was simultaneously recorded with the $\mathrm{CO}_{2}$ traces by introducing an additional pneumotachograph into the ventilation circuit. This allowed the analyses of volumetric capnograms in 20, 7, 32 and 9 patients in the Groups HL, $\mathrm{HC}, \mathrm{MC}$ and LC, respectively. The $15 \mathrm{~s} \mathrm{CO}_{2}$ and respiratory flow traces were imported into commercial signal analysis software (Biopac, Santa Barbara, CA, USA). Linear regression analysis was applied to obtain the slope of the third phase of the expiratory capnogram in the time domain $\left(\mathrm{S}_{\mathrm{T} \text {-III }}\right)$ and $\mathrm{CO}_{2}$ concentration was analyzed as a function of expired volume to obtain the volumetric third phase slope $\left(\mathrm{S}_{\mathrm{v}-\mathrm{III}}\right)$. These analyses were performed by fitting a line for each expiratory phase in the recordings in the linear phase before the end-expiratory peak $[6,11]$. Both $\mathrm{S}_{\mathrm{T} \text {-III }}$ and $\mathrm{S}_{\mathrm{V} \text {-III }}$ were normalized by dividing each slope by the average values of the corresponding $\mathrm{CO}_{2}$ concentration in mixed expired gas to obtain normalized time domain $\left(\mathrm{Sn}_{\mathrm{T} \text {-III }}\right)$ and volumetric $\left(\mathrm{Sn}_{\mathrm{V} \text {-III }}\right)$ third phase slopes $[2,21,22]$. Three to four expiratory traces were analyzed in each recording resulting in an ensemble averaging of 10 to 12 values under each condition.

\section{Measurement protocol}

The scheme of the experimental protocol is outlined in Figure 1. When stable hemodynamic and respiratory mechanical conditions had been reached while PEEP was maintained at $3 \mathrm{cmH}_{2} \mathrm{O}$, an arterial blood gas sample was taken, and dynamic compliance $(\mathrm{Crs})$ was recorded from the display of the respirator. The first capnogram trace was then collected followed by recording of the first Zrs data epoch. Two more capnographic and Zrs measurements were then made in alternating sequence at $60 \mathrm{~s}$ intervals. PEEP was next elevated to 6 and then $9 \mathrm{cmH}_{2} \mathrm{O}$, a 3 min equilibration period being permitted after each step, and the data collection procedure was repeated.

\section{Statistical analyses}

Scatters in measured variables are expressed as SE. The normality of the data was tested with the KolgomorovSmirnov test with Lilliefors correction. Two-way repeated measures analysis of variance (ANOVA) including an interaction term was used with the variables PEEP $(3,6$ and $9 \mathrm{cmH}_{2} \mathrm{O}$ ) and the group allocation (Groups HL, LC, $\mathrm{MC}$ and $\mathrm{HC}$ ) to establish the effects of lung volume and Crs on the respiratory mechanical, blood gas and capnographic variables. This statistical method was utilized to test the hypothesis that the level of Crs affects the PEEPdependent changes in the respiratory mechanical and 


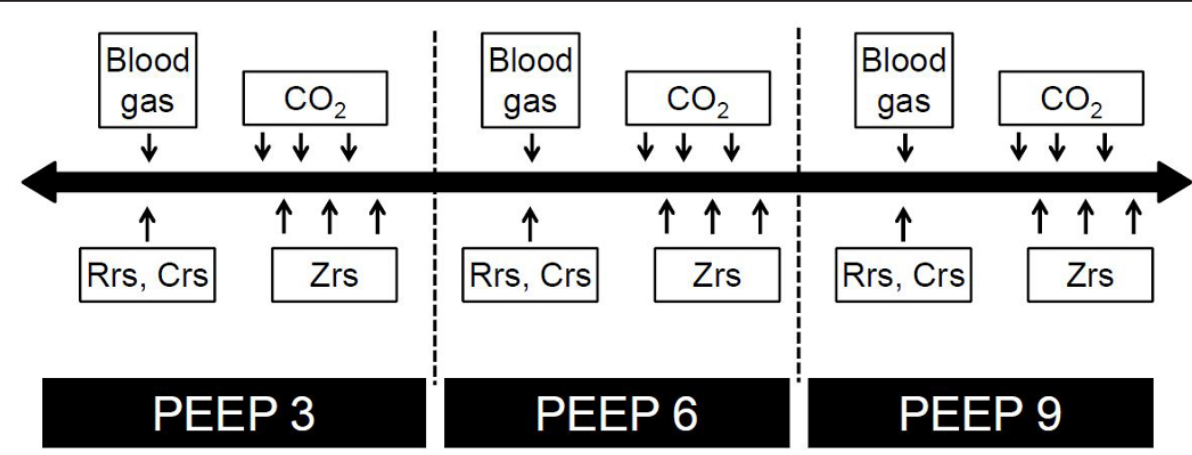

Figure 1 Timeline of the experimental protocol. Rrs and $\mathrm{Crs}$, readings from the respirator display; $\mathrm{CO}_{2}$, recording of capnogram curves; Zrs, forced oscillatory measurement of respiratory system impedance. $\mathrm{CO}_{2}$, carbon dioxide; $\mathrm{Crs}$, dynamic respiratory compliance; Rrs, total respiratory resistance; Zrs, input impedance of the respiratory system.

capnogram variables. Multiple linear regression analysis was performed to establish whether the levels of BMI and EF affect Crs. The Holm-Sidak multiple comparison procedure was adopted to compare the variables in the various study groups under different conditions. Chisquare test was used to assess whether there is a significant difference between the expected and the observed frequencies of gender, obesity, pulmonary and cardiac diseases in the protocol groups. The correlation between $\mathrm{S}_{\mathrm{T}-\mathrm{III}}$ and $\mathrm{S}_{\mathrm{V}-\mathrm{III}}$ were analyzed by Pearson test. The statistical tests were performed with a SigmaPlot statistical software package (Version 12, Systat Software, Inc. Chicago, IL, USA). All reported $P$ values are two-sided.

\section{Results}

The changes in the respiratory mechanical parameters, the partial pressure of arterial oxygen $\left(\mathrm{PaO}_{2}\right)$ and the indices obtained from the capnograms with increasing PEEP in the four groups of patients are depicted in Figure 2. The statistical analyses revealed significant interactions between the group allocation and PEEP, demonstrating that the respiratory compliance exerted significant effects on the responses to PEEP in the forced oscillatory mechanical parameters $(P<0.001$ for Raw, $\mathrm{G}$ and $\mathrm{H})$, for the Crs displayed by the respirator $(P<0.001), \mathrm{PaO}_{2}(P=$ $0.04)$, and the capnogram third phase slope variables $(P<$ 0.001 for $\mathrm{S}_{\mathrm{T}-\mathrm{III}}$ and $\mathrm{Sn}_{\mathrm{T}-\mathrm{III}}, P=0.003$ for $\mathrm{S}_{\mathrm{V}-\mathrm{III}}$, and $P=$ 0.002 and $\mathrm{Sn}_{\mathrm{V} \text {-III }}$ ). Time and volumetric capnogram variables exhibited similar Crs and PEEP dependences, which is also reflected in the significant correlations between $\mathrm{S}_{\mathrm{T} \text {-III }}$ and $\mathrm{S}_{\mathrm{V} \text {-III }}$ in Groups HL $(\mathrm{R}=0.4, P=0.002)$, HC $(\mathrm{R}=0.79, P<0.001), \mathrm{LC}(\mathrm{R}=0.45, P=0.02)$ and $\mathrm{MC}(\mathrm{R}=$ $0.79, P<0.001)$.

The greatest Raw, G, $\mathrm{H}$ and the lowest $\mathrm{PaO}_{2}$ were observed for the patients in Group LC, and these patients generally exhibited the greatest response to PEEP. The patients in Group MC still exhibited elevated Raw, G and $\mathrm{H}$ with a more moderate, but still significant response to
PEEP changes. The lowest forced oscillatory airway and tissue parameters and the greatest $\mathrm{PaO}_{2}$ were obtained in the patients in Groups HL and HC, and their changes with PEEP were generally mild. The capnogram third phase indices were highest in Group $\mathrm{HC}$ and somewhat lower in Group MC, with both groups exhibiting marked decreases with increasing PEEP. The variables characterizing the third phase slopes from the capnogram were lowest in the patients in Group HL.

Figure 3 depicts the relationship of Raw and $\mathrm{S}_{\mathrm{T}-\mathrm{III}}$ in the individual patients and the group means for the four protocol groups following the increases of PEEP. In all patients, Raw and $S_{\text {T-III }}$ underwent concomitant monotonous decreases with increasing PEEP, but marked differences were observed between the protocol groups in the relationships of these parameters. The marked decreases in the high initial Raw values were associated with substantially smaller drops in $S_{\text {III }}$ in the patients in Group LC, whereas the PEEP-induced decreases in $\mathrm{S}_{\mathrm{III}}$ were more pronounced than those in Raw in the patients in Group HC. The patients in Group MC exhibited an intermediate Raw-S $\mathrm{S}_{\mathrm{T}-\mathrm{III}}$ relationship. This trend of association was observed in the patients in Group HL at markedly lower levels of Raw and $\mathrm{S}_{\mathrm{III}}$.

To examine the possible roles of obesity and lung congestion in the increased level of Crs, the effects of BMI and EF were considered (Figure 4). The patients in Group LC had significantly higher BMI $(P<0.001)$ and/or lower EF $(P<0.001)$ than those in Groups HL or $\mathrm{HC}$, indicating that the low Crs was a consequence of restrictive changes resulting from obesity and/or heart failure leading to pulmonary congestion (multiple linear regression coefficient of $R=0.58$ ). The important effects of BMI and EF on the group allocation was confirmed by the presence of a significant correlation $(\mathrm{R}=0.53, P=0.005$ and $P<0.0001$ for EF and BMI, respectively). 

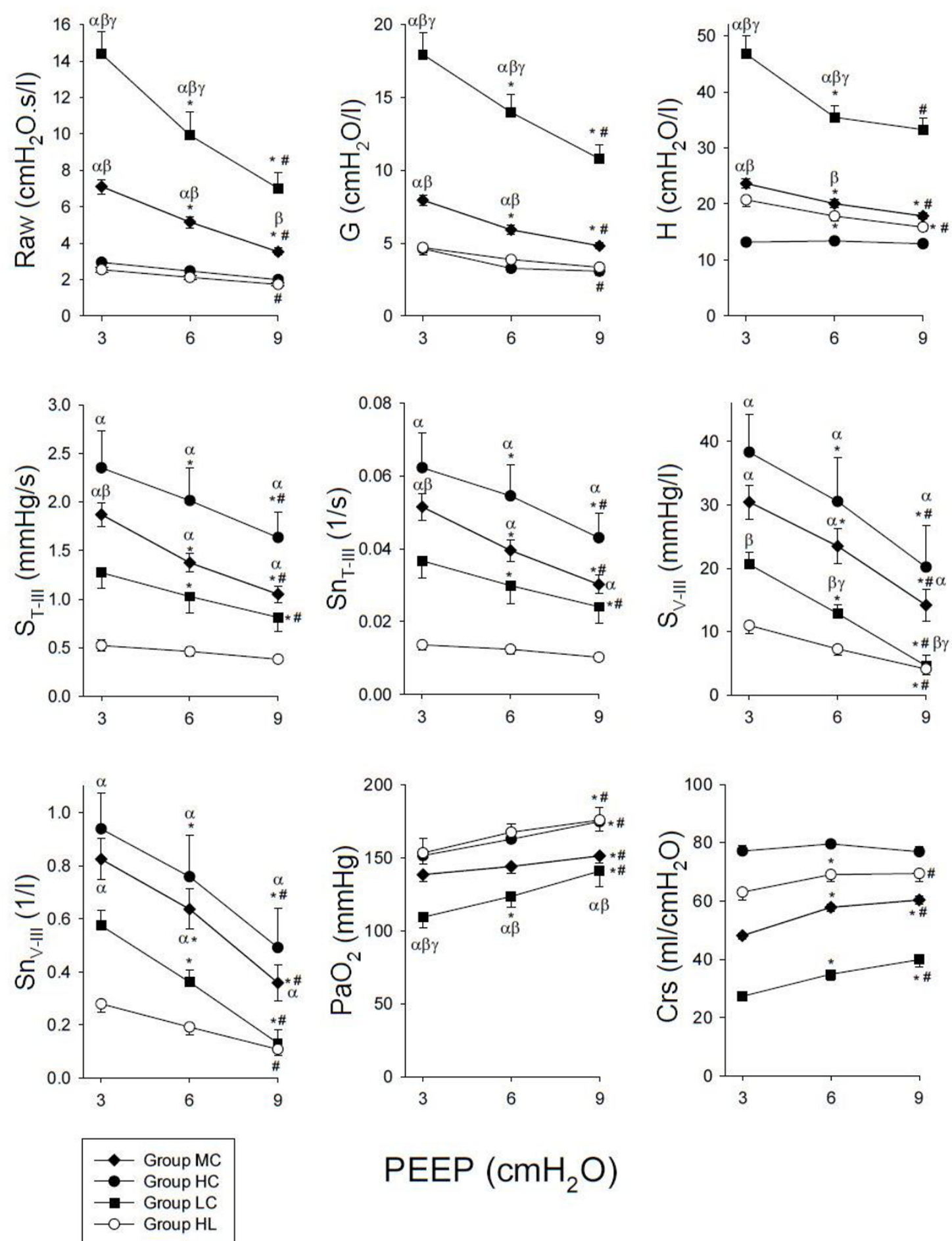

Figure 2 Forced oscillatory airway (Raw, airway resistance) and respiratory tissue (G, damping and $H$, elastance) mechanical parameters, the slope of the third phase of the capnogram as expressed in the time domain before $\left(\mathrm{S}_{\mathrm{T}-\text {-III }}\right)$ and after normalization for the mean expired $\mathrm{CO} 2\left(\mathrm{Sn}_{\mathrm{T}-\mathrm{III}}\right)$, or as a function of expired volume before $\left(\mathrm{S}_{\mathrm{V}-\mathrm{III}}\right)$ and after $\left(\mathrm{Sn}_{\mathrm{V}-\mathrm{III}}\right)$ normalization, partial pressure of oxygen in the arterial blood $\left(\mathrm{PaO}_{2}\right)$ and dynamic compliance (Crs) displayed by the respirator in patients with healthy lungs (Group $\mathrm{HL}$ ), and in patients with respiratory symptoms with Crs in the lowest tenth percentile (Group LC), with Crs between the tenth and the ninetieth percentile (Group MC) and with Crs above the ninetieth percentile in (Group HC). ${ }^{*}, P<0.05$ vs. the variable at the previous PEEP level; $\#, P<0.05$ vs. a variable at a PEEP of $3 \mathrm{cmH}_{2} \mathrm{O} . \alpha, P<0.05$ vs. Group HL within a PEEP; $\beta, P<0.05$ vs. Group HC within a PEEP; $\gamma$, $P<0.05$ vs. Group MC within a PEEP. 


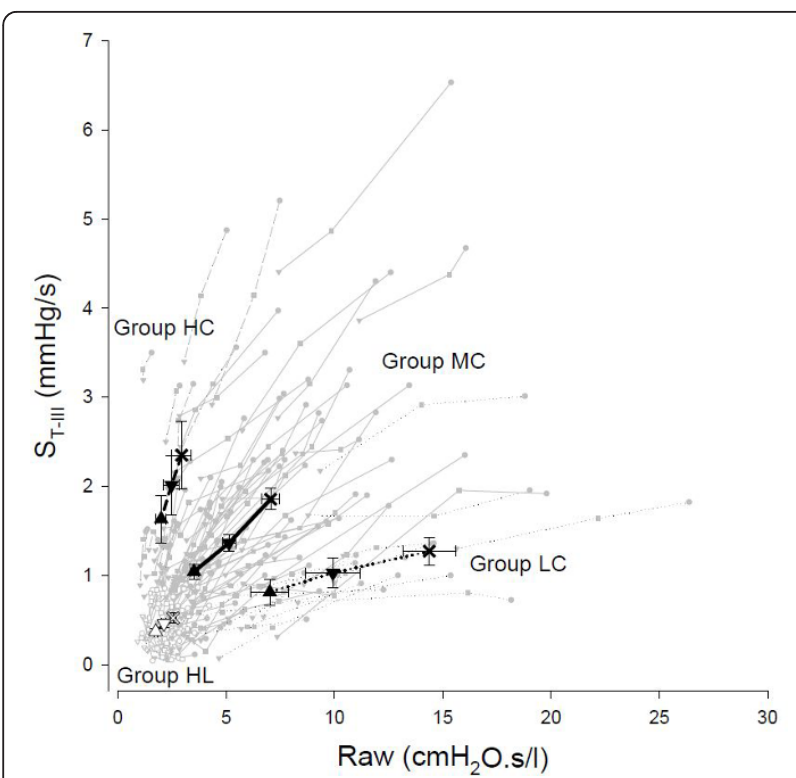

Figure 3 Relationship between forced oscillatory airway resistance (Raw) and phase III slope of time capnogram $\left(\mathrm{S}_{\mathrm{T}-\mathrm{III}}\right)$ at PEEP levels of $3(\mathrm{X}), 6(\nabla)$ and $9 \mathrm{~cm} \mathrm{H}_{2} \mathrm{O}(\Delta)$ in patients with healthy lungs (Group $\mathrm{HL}$ ), and in patients with respiratory symptoms with dynamic respiratory compliance (Crs) in the lowest tenth percentile (Group LC), with Crs between the tenth and ninetieth percentile (Group MC) and with Crs above the ninetieth percentile (Group HC). Thin grey lines denote individual patients; thick black lines with symbols show group mean and SE values.

\section{Discussion}

The changes in airway and respiratory tissue mechanics were compared with capnogram third phase indices in a relatively large cohort of patients to clarify its monitoring value in mechanically ventilated patients. Capnogram third phase slopes expressed in the time domain or as a function of expired volume exhibited similar PEEP dependencies and distribution between the protocol groups. Detailed analysis of the time capnogram revealed a strong association between Raw and $S_{\mathrm{T} \text {-III }}$ when the respiratory mechanics was altered by increasing PEEP, which was significantly affected by the degree of expiratory driving pressure of the respiratory system. Accordingly, grouping of the patients based on their Crs revealed that i) the decrease in Raw with increasing PEEP was reflected in a sharp decrease in $\mathrm{S}_{\mathrm{T} \text {-III }}$ in patients with low $\mathrm{Crs}$, ii) the increase in airway diameter with increasing PEEP was still reflected in a pronounced decrease in $\mathrm{S}_{\mathrm{T}-\mathrm{III}}$ in patients with intermediate Crs, and iii) $\mathrm{S}_{\mathrm{T}-\mathrm{III}}$ was insensitive to changes in airway caliber when the Crs was high.

The extent of emptying of lung compartments containing various $\mathrm{CO}_{2}$ concentrations during mechanical ventilation and the shape of the resulting capnogram are determined by the airway geometry (that is, the resistance) and the elastic recoil of the respiratory tissues (that is, the driving pressure). Whereas the former has been investigated extensively $[4,6,12,13,15]$, the importance of the latter factor remained unknown. While the time capnogram is most commonly used in clinical practice during mechanical ventilation, distinction of the second and third phases is not always trivial from the time domain analyses and this approach also excludes the consideration of the absolute concentration of $\mathrm{CO}_{2}$ in the expired gas. Therefore, we also performed volumetric capnography in a subgroup of patients and normalized the capnogram third phase slope. The similar picture of these different slopes and the significant correlation between them demonstrates that the $\mathrm{S}_{\mathrm{T}-\mathrm{III}}$ used in clinical practice can provide relevant information about lung emptying. With the aim of acquiring a general picture, the bedside Crs was used in the present study to group the patients. A strong correlation was earlier demonstrated between the respiratory elastance derived from $\mathrm{H}$ and the $\mathrm{Crs}$ [23], which justifies the choice of $\mathrm{Crs}$ as an appropriate indicator of the respiratory recoil.

We formed four groups with regard to the clinical symptoms (healthy lungs) and the Crs values (diseased lungs with low, medium or high $\mathrm{Crs}$ ). As expected, the variables reflecting resistive behavior were lowest in the Group HL and they had intermediate $\mathrm{H}$ and Crs, permitting fast emptying of the relatively homogeneous lungs, which then results in low capnogram third phase slope, and good $\mathrm{PaO}_{2}$ (Figure 2). The increase of PEEP to $6 \mathrm{cmH}_{2} \mathrm{O}$ caused no further improvement. The slight, but significant decreases in Raw and $\mathrm{H}$ and increases in Crs and $\mathrm{PaO}_{2}$ at PEEP $9 \mathrm{cmH}_{2} \mathrm{O}$ may be a consequence of lung recruitment. The lack of decrease in the third phase slope indicates that this opening was relatively uniform in the lung periphery.

The patients in Group $\mathrm{HC}$ exhibited similar resistive properties to those in Group HL. However, the high Crs and low $\mathrm{H}$ may be a consequence of the loss of elastic recoil in the respiratory tissues, most probably due to emphysematous destruction, which was present in the vast majority of the patients in this group (Table 1 ). The presence of ventilation heterogeneities is also apparent from the highest third phase slope indices. These can be explained by the existence of peripheral lung units with different small airway calibers and local time constants, resulting in a heterogeneous working lung. This structure leads to gas compartments containing variable $\mathrm{CO}_{2}$ concentrations and also results in different local expiratory flows [11]. These phenomena contribute to the sequential emptying of the lung periphery in time, which then increases the time domain and volumetric $S_{\text {III }}$ values $[3-5,10,24]$. Since Raw reflects mainly the flow resistance of the central conducting airways $[19,23,25]$, this parameter is not able to detect such alterations in the presence of emphysematous changes [26]. This pathology 

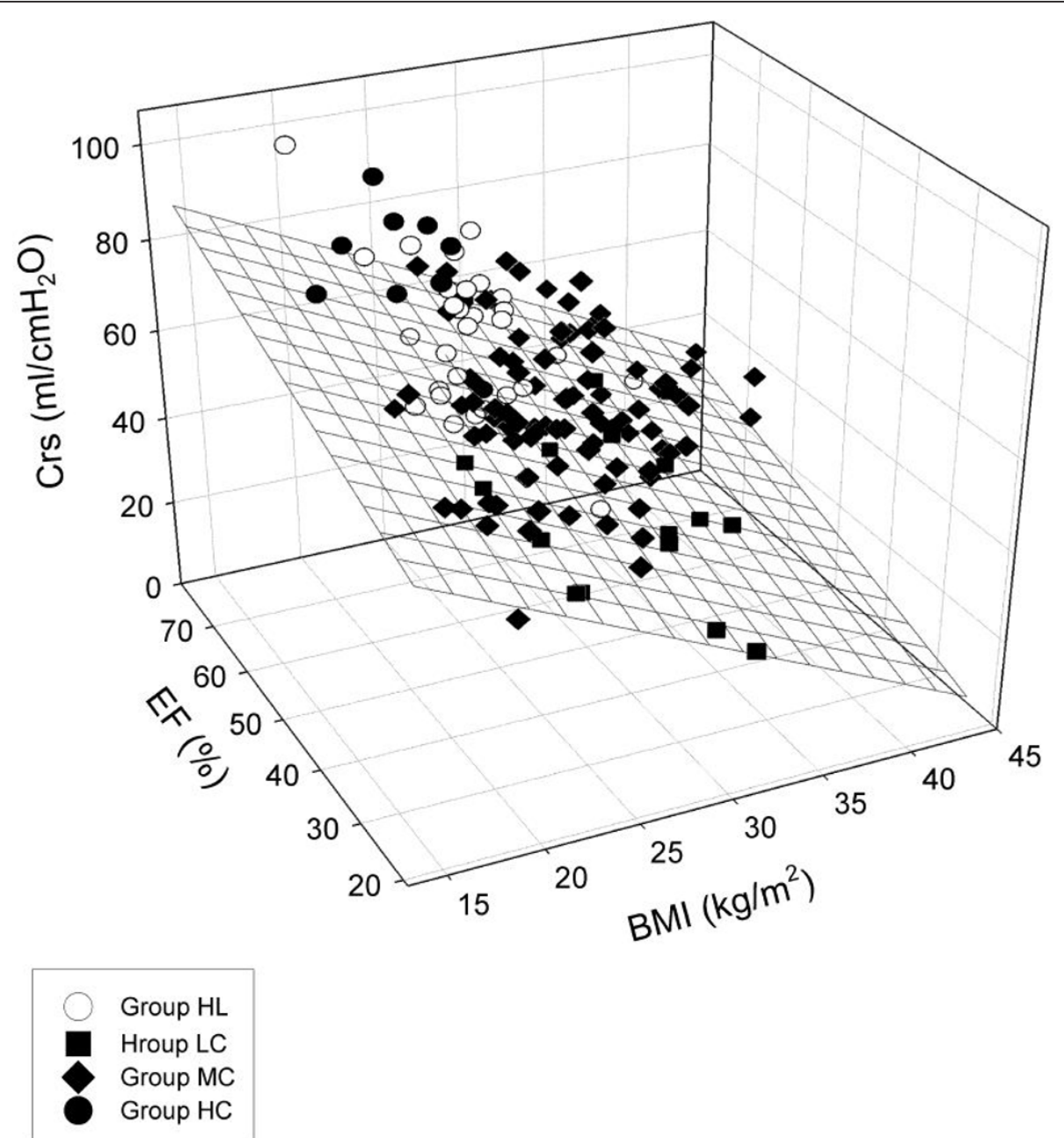

Figure 4 Effects of body mass index (BMI) and ejection fraction (EF) on dynamic respiratory compliance (Crs). The best fit plane is demonstrated with a mesh surface.

diminishes predominantly the expiratory flow, while the filling of the lung during mechanical ventilation may remain unaffected or even increased, explaining why $\mathrm{PaO}_{2}$ was close to normal. The elevation of PEEP in Group HC decreased G, which is a prerequisite of decreased ventilation heterogeneities with alveolar recruitment $[27,28]$, reflecting in lower capnogram third phase slopes, $\mathrm{H}$, higher $\mathrm{Crs}$ and better $\mathrm{PaO}_{2}$ [12].

The worst respiratory mechanics and the lowest $\mathrm{PaO}_{2}$ were observed in the patients in Group LC. Despite this striking difference, $\mathrm{S}_{\mathrm{III}}$ expressed in time or by volumetry did not differ significantly from those observed in Group HL. This leads to the important observation that even hypoxemia may be associated with a medium level of capnogram third phase slopes, which corresponds to the limited value of capnometry in the assessment of adequate blood oxygenation in this pathology $[29,30]$. These results can most probably be attributed to the presence of lung regions that remain closed throughout the entire ventilator cycle, leading to some relatively open and fairly uniform working lung units and other, permanently closed, atelectatic lung units. In other words, the closing capacity in these lungs is expected to be higher than the sum of the functional residual capacity and the tidal volume. The persistent lung volume loss with subsequent decrease in the overall airway cross-sectional area is probably reflected in the substantially elevated Raw. Since PEEP elevation may be able to reopen these atelectases, the involvement of these phenomena is substantiated by the most pronounced decreases in the mechanical parameters with increasing PEEP resulting in lower $\mathrm{S}_{\mathrm{III}}$ and elevated $\mathrm{PaO}_{2}$, which corresponds to earlier results on similar stiff lungs [10]. Our results confirm previous clinical observations $[9,12,25]$ that this pathophysiology can be triggered by obesity and/ or lung congestion arising from a poor EF (Table 1). Taking into account the individual and the combined 
effects of BMI and EF revealed that low EF or high BMI themselves may be responsible for the compromised Crs. However, the combination of such pathologies exerts additional detrimental effects that lower $\mathrm{Crs}$ even more dramatically (Figure 4).

Group MC comprised patients with pulmonary pathologies with an intermediate Crs, a cohort that can be characterized by somewhat elevated airway and respiratory tissue parameters, and ventilation heterogeneities reflected in abnormally high capnography slope characteristics at a PEEP of $3 \mathrm{cmH}_{2} \mathrm{O}$. This variable and the intermediate response to PEEP can be explained by concomitant presence of phenomena existing in Groups $\mathrm{HC}$ and $\mathrm{LC}$, that is, combined effects of expiratory flow limitation and persistent atelectases.

The overall Raw-S $\mathrm{S}_{\mathrm{T}-\mathrm{III}}$ relationship was not strong enough to predict the value of Raw from $S_{\text {T-III }}$ (Figure 3), in agreement with previous findings $[6,18]$. However, the changes in $\mathrm{S}_{\mathrm{T} \text {-III }}$ within an individual patient were appropriate for an assessment or revealing trends of the altered Raw. It should be noted that the Raw-S $\mathrm{S}_{\mathrm{T}-\mathrm{III}}$ relationship within a patient was highly dependent on the elastic recoil of the respiratory system. In the case of a small Crs, a minor change in $\mathrm{S}_{\mathrm{T}-\mathrm{III}}$ may reflect major alterations in airway patency. In contrast, large alterations in $\mathrm{S}_{\mathrm{T}-\mathrm{III}}$ may still be associated with small variations in Raw if Crs is high. This finding may explain the controversy in the literature concerning the presence or absence of a correlation between lung function parameters and capnogram indices $[11,13,15,16]$.

The limitations of this study relate to the possible presence of complex cardiopulmonary pathologies within a given patient. The coexistence of opposing factors such as emphysematous changes and a poor left ventricular function precludes identification of the individual effects of pulmonary diseases on the course of the capnogram. An additional aspect is that the surgery did not allow a more time-consuming randomization of the PEEP levels. However, care was taken to provide sufficient time following a change in conditions so that equilibrium was reached, similarly to that allowed following PEEP changes in severe COPD patients [27]. Another methodological limitation is related to the complex effects of PEEP including modification of the lung perfusion [2], increased functional residual capacity $[21,31]$, which may all bias the changes in $S_{\text {III }}$ and/or the mechanical parameters. However, our results are consistent even on PEEP $3 \mathrm{cmH}_{2} \mathrm{O}$ alone and the PEEP changes can be considered as reinforcement of the results and mechanisms that existed already at the lower PEEP. Auto-PEEP may be another important factor imposing a potential error with this bias being the most apparent in the patients with high Raw (that is, Group LC) or low driving pressure and compromised emptying of emphysematous destructed alveoli (that is, Group HC). Excluding the auto-PEEP would even enhance the Raw dependence with PEEP, since Raw would theoretically be even higher if auto-PEEP would have been ruled out. Another important feature of the present study is the use of Crs to separate the study groups. Since this parameter incorporates lung and chest wall properties, a separate assessment of which of these compartments are responsible for the altered elastic recoil of the respiratory system is not possible.

\section{Conclusions}

In summary, measurement of the respiratory mechanics and analysis of the capnogram slope demonstrated that changes in $\mathrm{S}_{\mathrm{III}}$ expressed in time or by volumetry provide useful information concerning alterations in airway caliber, but only within an individual patient. The assessment of $\mathrm{S}_{\mathrm{T} \text {-III }}$ during mechanical ventilation may be of value for bedside monitoring of the airway resistance, but its sensitivity depends on the elastic recoil of the respiratory system. $\mathrm{S}_{\mathrm{T}-\mathrm{III}}$ exhibits high sensitivity to detect changes in the airway resistance in case of high Crs, when the lung emptying is governed primarily by the small airway and alveolar geometry. In cases of stiff respiratory tissues, however, $\mathrm{S}_{\mathrm{T} \text {-III }}$ displays low sensitivity in indicating changes in airway caliber, when the lung emptying is determined by the high elastic recoil and depends less on the small airway geometry. The relatively low $\mathrm{S}_{\mathrm{T} \text {-III }}$ may coincide with the compromised $\mathrm{PaO}_{2}$ in these patients, which suggests that a low $\mathrm{S}_{\mathrm{T}-\mathrm{III}}$ does not predict appropriate oxygenation. Thus, the shape of the capnogram should always be evaluated bedside in conjunction with Crs. The joint assessment of the capnogram and the respiratory mechanics is of particular importance in clinical situations when patients with a high BMI and/or a compromised left ventricular function are anesthetized and ventilated.

\section{Key messages}

- The phase III slope of the capnogram evaluated in the time domain or by volumetry exhibits similar PEEP dependencies and distribution between the protocol groups formed on the basis of Crs.

- Crs significantly affects the sensitivity of the phase III slope of the capnogram in the time domain $\left(\mathrm{S}_{\mathrm{T}}\right.$ III) and by volumetry to airway dimensions.

- $\mathrm{S}_{\mathrm{T} \text {-III }}$ detects changes in the airway resistance sensitively in cases of high Crs.

- $\mathrm{S}_{\mathrm{T} \text {-III }}$ displays low sensitivity in cases of stiff respiratory tissues, in indicating changes in airway caliber.

- In conclusion, assessment of the capnogram shape should always be coupled with Crs when the airway resistance or oxygenation are evaluated. 


\section{Abbreviations}

BMI: body mass index; $\mathrm{CO}_{2}$ : carbon dioxide; $\mathrm{COPD}$ : chronic obstructive pulmonary disease; Crs: dynamic respiratory compliance; EF: ejection fraction; $\mathrm{FiO}_{2}$ : fraction of inspired oxygen; G: respiratory tissue damping; Group HC: group of patients with high dynamic respiratory compliance; Group HL: group of patients with healthy lungs; Group LC: group of patients with low dynamic respiratory compliance; Group MC: group of patients with medium dynamic respiratory compliance; $\mathrm{H}$ : respiratory tissue elastance; law: airway inertance; Pao: airway opening pressure; $\mathrm{PaO}_{2}$ : partial pressure of arterial oxygen; PEEP: positive end-expiratory pressure; Raw: airway resistance; Rrs: total respiratory resistance; $\mathrm{S}_{\| I}$ : slope of phase III of the capnogram; $\mathrm{Sn}_{\mathrm{T}-\|}$ : normalized third phase slope of the expiratory capnogram in the time domain; $S_{\text {V-III: }}$ normalized volumetric third phase slope of the expiratory capnogram; $\mathrm{S}_{\mathrm{T}-\| 1}$ : third phase slope of the expiratory capnogram in the time domain; $\mathrm{S}_{\mathrm{v}-111}$ : volumetric third phase slope of the expiratory capnogram; $\mathrm{V}^{\prime}$ : tracheal airflow; Zrs: input impedance of the respiratory system.

\section{Acknowledgements}

This work was supported by Hungarian Basic Scientific Research Grant OTKA K81179. Ferenc Peták is supported by a Bolyai János Research Fellowship. The authors thank Prof. Zoltán Hantos for his highly pertinent advice and for his contribution in initiating volumetric measurements during the study.

\section{Author details}

'Department of Anesthesiology and Intensive Therapy, University of Szeged, 6 Semmelweis u., H-6720 Szeged, Hungary. ${ }^{2}$ Department of Medical Physics and Informatics, University of Szeged, 9 Korányi fasor, H-6720 Szeged, Hungary. ${ }^{3}$ Department of Anesthesiology, German Heart Center, 36 Lazarettstr., D-80636 Munich, Germany. ${ }^{4}$ Department of Cardiac Surgery, University of Szeged, 4 Pécsi u., H-6720 Szeged, Hungary.

\section{Authors' contributions}

BB conducted the design of the study and had a major role in data collection and drafting the manuscript. CsZs and PNM helped with the data collection and analyses. BG performed the surgical preparation and helped with the measurements. CD participated in the study design, data collection and helped with processing the data. FP supervised the data collection and analyses, contributed to the development of the study design and in the manuscript preparation. All authors read and approved the final manuscript.

\section{Competing interests}

The authors declare they have no competing interests in relation to this manuscript.

Received: 27 April 2012 Revised: 6 September 2012

Accepted: 2 October 2012 Published: 2 October 2012

\section{References}

1. Dubois $A B$, Britt $A G$, Fenn WO: Alveolar $\mathrm{CO} 2$ during the respiratory cycle. J Appl Physiol 1952, 4:535-548.

2. Tusman G, Areta M, Climente C, Plit R, Suarez-Sipmann F, RodriguezNieto MJ, Peces-Barba G, Turchetto E, Bohm SH: Effect of pulmonary perfusion on the slopes of single-breath test of CO2. J Appl Physiol 2005, 99:650-655.

3. Crawford AB, Makowska M, Paiva M, Engel LA: Convection- and diffusiondependent ventilation maldistribution in normal subjects. J Appl Physiol 1985, 59:838-846.

4. Stromberg NO, Gustafsson PM: Ventilation inhomogeneity assessed by nitrogen washout and ventilation-perfusion mismatch by capnography in stable and induced airway obstruction. Pediatr Pulmonol 2000, 29:94-102.

5. Dutrieue B, Vanholsbeeck F, Verbanck S, Paiva M: A human acinar structure for simulation of realistic alveolar plateau slopes. J Appl Physiol 2000, 89:1859-1867.

6. Blanch L, Lucangelo U, Lopez-Aguilar J, Fernandez R, Romero PV: Volumetric capnography in patients with acute lung injury: effects of positive end-expiratory pressure. Eur Respir J 1999, 13:1048-1054.

7. Romero PV, Rodriguez B, de Oliveira D, Blanch L, Manresa F: Volumetric capnography and chronic obstructive pulmonary disease staging. Int J Chron Obstruct Pulmon Dis 2007, 2:381-391.
8. Veronez L, Moreira MM, Soares ST, Pereira MC, Ribeiro MA, Ribeiro JD, Terzi RG, Martins LC, Paschoal IA: Volumetric capnography for the evaluation of pulmonary disease in adult patients with cystic fibrosis and noncystic fibrosis bronchiectasis. Lung 2010, 188:263-268.

9. Hoffbrand BI: The expiratory capnogram: a measure of ventilationperfusion inequalities. Thorax 1966, 21:518-523.

10. Tusman G, Suarez-Sipmann F, Bohm SH, Borges JB, Hedenstierna G: Capnography reflects ventilation/perfusion distribution in a model of acute lung injury. Acta Anaesthesiol Scand 2011, 55:597-606.

11. Krauss B, Deykin A, Lam A, Ryoo JJ, Hampton DR, Schmitt PW, Falk JL: Capnogram shape in obstructive lung disease. Anesth Analg 2005, 100:884-888.

12. Bohm SH, Maisch S, von Sandersleben A, Thamm O, Passoni I, Martinez Arca J, Tusman G: The effects of lung recruitment on the Phase III slope of volumetric capnography in morbidly obese patients. Anesth Analg 2009, 109:151-159.

13. Nik Hisamuddin NA, Rashidi A, Chew KS, Kamaruddin J, Idzwan Z, Teo AH: Correlations between capnographic waveforms and peak flow meter measurement in emergency department management of asthma. Int J Emerg Med 2009, 2:83-89.

14. Thompson JE, Jaffe MB: Capnographic waveforms in the mechanically ventilated patient. Respir Care 2005, 50:100-108, discussion 108-109.

15. Yaron M, Padyk P, Hutsinpiller M, Cairns CB: Utility of the expiratory capnogram in the assessment of bronchospasm. Ann Emerg Med 1996, 28:403-407.

16. You B, Peslin R, Duvivier C, Vu VD, Grilliat JP: Expiratory capnography in asthma: evaluation of various shape indices. Eur Respir J 1994, 7:318-323.

17. Kars AH, Bogaard JM, Stijnen T, de Vries J, Verbraak AF, Hilvering C: Dead space and slope indices from the expiratory carbon dioxide tensionvolume curve. Eur Respir J 1997, 10:1829-1836.

18. Blanch L, Fernandez R, Saura P, Baigorri F, Artigas A: Relationship between expired capnogram and respiratory system resistance in critically ill patients during total ventilatory support. Chest 1994, 105:219-223.

19. Babik B, Asztalos T, Petak F, Deak ZI, Hantos Z: Changes in respiratory mechanics during cardiac surgery. Anesth Analg 2003, 96:1280-1287.

20. Hantos Z, Daroczy B, Suki B, Nagy S, Fredberg JJ: Input impedance and peripheral inhomogeneity of dog lungs. J Appl Physiol 1992, 72:168-178.

21. Ream RS, Schreiner MS, Neff JD, McRae KM, Jawad AF, Scherer PW, Neufeld GR: Volumetric capnography in children. Influence of growth on the alveolar plateau slope. Anesthesiology 1995, 82:64-73.

22. Tsoukias NM, Tannous Z, Wilson AF, George SC: Single-exhalation profiles of $\mathrm{NO}$ and $\mathrm{CO} 2$ in humans: effect of dynamically changing flow rate. J Appl Physiol 1998, 85:642-652.

23. Babik B, Petak F, Asztalos T, Deak ZI, Bogats G, Hantos Z: Components of respiratory resistance monitored in mechanically ventilated patients. Eur Respir J 2002, 20:1538-1544.

24. van Meerten RJ: Expiratory gas concentration curves for examination of uneven distribution of ventilation and perfusion in the lung. First communication: theory. Respiration 1970, 27:552-564.

25. Albu G, Babik B, Kesmarky K, Balazs M, Hantos Z, Petak F: Changes in airway and respiratory tissue mechanics after cardiac surgery. Ann Thorac Surg 2010, 89:1218-1226.

26. Tolnai J, Szabari MV, Albu G, Maar BA, Parameswaran H, Bartolak-Suki E, Suki B, Hantos Z: Functional and morphological assessment of early impairment of airway function in a rat model of emphysema. J Appl Physiol 2012, 112:1932-1939.

27. Lorx A, Szabo B, Hercsuth M, Penzes I, Hantos Z: Low-frequency assessment of airway and tissue mechanics in ventilated COPD patients. J Appl Physiol 2009, 107:1884-1892.

28. Lutchen KR, Hantos Z, Petak F, Adamicza A, Suki B: Airway inhomogeneities contribute to apparent lung tissue mechanics during constriction. J Appl Physiol 1996, 80:1841-1849.

29. Napolitano LM: Capnography in critical care: accurate assessment of ARDS therapy? Crit Care Med 1999, 27:862-863.

30. Morley TF, Giaimo J, Maroszan E, Bermingham J, Gordon R, Griesback R, Zappasodi SJ, Giudice JC: Use of capnography for assessment of the adequacy of alveolar ventilation during weaning from mechanical ventilation. Am Rev Respir Dis 1993, 148:339-344.

31. Tusman G, Bohm SH, Suarez-Sipmann F, Scandurra A, Hedenstierna G: Lung recruitment and positive end-expiratory pressure have different effects 
on $\mathrm{CO} 2$ elimination in healthy and sick lungs. Anesth Analg 2010, 111:968-977.

doi:10.1186/cc11659

Cite this article as: Babik et al.: Effects of respiratory mechanics on the capnogram phases: importance of dynamic compliance of the respiratory system. Critical Care 2012 16:R177.

Submit your next manuscript to BioMed Central and take full advantage of:

- Convenient online submission

- Thorough peer review

- No space constraints or color figure charges

- Immediate publication on acceptance

- Inclusion in PubMed, CAS, Scopus and Google Scholar

- Research which is freely available for redistribution 